\title{
Secrets of omega-3 oil
}

\section{Suvarna BS}

Assistant Professor, Department of Pharmacology, Melaka Manipal Medical College (ICHS), Manipal, India

$\mathrm{T}_{\mathrm{cos}}^{\mathrm{h}}$ here are good and bad fats. The Omega-3 is considered to be a good fat ${ }^{1}$. Omega- 3 fatty acids are considered essential fatty acids, which means that they are essential to human health but cannot be manufactured by the body. For this reason, omega-3 fatty acids must be obtained from food. Omega-3 fatty acids can be found in fish and certain plant oils and hence are the primary dietary source of omega-3 fatty acids. Another potential source of omega-3 fatty acids is New Zealand green lipped mussels (Perna canaliculus) ${ }^{2}$, used for centuries by the Maories to promote good health. Polyunsaturated fatty acids (PUFAs), omega-3 and omega- 6 fatty acids play a crucial role in brain function as well as normal growth and development. It is important to maintain an appropriate balance of omega-3 and omega- 6 (another essential fatty acid) in the diet as these two substances work together to promote health. Omega oil's excellent levels of precursor raw material fatty acids Alpha-linolenic acid (ALA) - Omega 3 and the precursor linolenic acid (LA) - Omega 6, make it an ideal supplement for those whose lifestyle makes them suffer the consequences of deficiency in the in taking of fatty acid products that are essential for a smooth running metabolism. Our body requires an ideal balance between Omega $3 \& 6$ to maintain its correct functioning. Eicosapentaenoic acid (EPA) and Docosahexaenoic acid (DHA) are found in cold-water fish such as salmon, mackerel, halibut, sardines, and herring. ALA is found in flaxseeds, flaxseed oil, canola (rapeseed) oil, soybeans, soybean oil, pumpkin seeds, pumpkin seed oil, purslane, perilla seed oil, walnuts, and walnut oil.

There are three major types of omega 3 fatty acids that are ingested in foods and used by the body: ALA, EPA, and DHA. Once eaten, the body converts ALA to EPA and DHA, the two types of omega-3 fatty acids more readily used by the body.

There have been a lot of studies conducted on Omega -3 oil. They have been tried in various conditions and diseases and proved to be effacious.

\section{Central nervous system}

\section{Manic/Depression (Bipolar Disorder)}

The omega-3 fatty acids are important components of nerve cell membranes. They help nerve cells communicate with each other, which is an essential step in maintaining good mental health. People who do not get enough omega- 3 fatty acids or do not maintain a healthy balance of omega- 3 to omega- 6 fatty acids in their diet may be at an increased risk for depression. In a study with bipolar disorder, those who were treated with EPA and DHA (in combination with their usual mood stabilizing medications) for four months experienced fewer mood swings and recurrence of either depression or mania than those who received placebo ${ }^{3}$. A similar but larger study is currently underway at the University Of California- Los Angeles School Of Medicine 4 . Harvard Medical School has successfully used fish oil supplements to treat bipolar disorder (manic-depressive illness). Omega 3 has also encouraging results in the treatment of schizophrenia. In a study of people with depression, those who ate a healthy diet consisting of fatty fish two to three times per week for 5 years experienced a significant reduction in feelings of depression and hostility. But there are few controversies regarding omega-3 uses in schizophrenia. Preliminary evidence $^{5}$ suggests that people with schizophrenia experience an improvement in symptoms when given omega-3 fatty acids. However, a recent well-designed study ${ }^{6}$ concluded that EPA supplements are no better than placebo in improving symptoms of this condition. The conflicting results suggest that more research is needed before conclusions can be drawn about the benefit of omega-3 fatty acids for schizophrenia. Similar to diabetes, people with schizophrenia may not be able to convert ALA to EPA or DHA efficiently ${ }^{6,7}$. A recent study found that Alzheimer's patients given an Omega-3 rich supplement experienced a significant improvement in their quality of life.

\section{Attention deficit hyperactivity disorder}

Other alarming evidence is that a low level of omega 3 in children is associated with hyperactivity. The latest findings ${ }^{8}$, from the University of Adelaide, are the first to show that omega-3 fish oil may be better than medication

\footnotetext{
Correspondence

Dr. Beena S Suvarna

Asst Prof, Dept of Pharmacology

Melaka Manipal Medical College (ICHS),

Manipal-576104, India

E-mail: drbsusheel@yahoo.co.uk
} 
at treating Attention Deficit Hyperactivity Disorder (ADHD). A daily dose of fish oil is better at treating hyperactivity. Just six capsules a day of the naturallyoccurring oil can vastly improve the brain power and concentration of hyperactive children. The Australian researchers looked at the effect fish oil capsules on a group 7 to 12-year-olds with ADHD. Some were given the capsules, while others took a placebo or dummy capsule. Within three months, the behaviour of those on the fish oils had dramatically improved and, by seven months, many of the children were less restless and doing better at school. The most striking improvement was in concentration or attention, which improved by a third. When those taking the dummy capsules switched to the fish oils, they also saw their behaviour improve. The studies also showed children became calmer and were doing better at school and were able to concentrate longer and were therefore reading more. 'Overall, 30 to 40 per cent of children over 15 weeks and 40 to 50 per cent of children over 30 weeks had improvements.' Week-on-week improvements were still being seen at the end of the study - suggesting the results of an even longer course of treatment, may be even more dramatic. "Fish and seafood are the only ready-made sources of the essential Omega-3 fatty acids EPA and DHA which we need for our brains," explains Dr Alex Richardson, a neuroscientist based at Oxford University. "If we don't get them from fish, we have to build them from other, simpler forms of the fatty acids found in green leafy vegetables and some nuts and seeds.

The exact mechanism of why fish oils help the brain is still being studied in depth", but they do. "Parents should choose a fish oil that is high in EPA, rather than high in DHA. Fish oil supplements should not be confused with cod liver oil supplements which, when taken at the high doses needed to help with dyslexia and ADHD, may actually cause an overdose of Vitamin $\mathrm{A}$ in the body. It is also more likely that the liver of the fish will contain more pollutants and toxins than the oils from the body of the fish. Parents wondering if their children have a deficiency of fatty acids, the signs are dry and lackluster skin and hair, dry bumpy skin at the top of the arms and weak, brittle nails. In animal studies, low levels of omega-3 fatty acids have been shown to lower the concentration of certain brain chemicals (such as dopamine and serotonin) related to attention and motivation. Studies that examine the ability of omega-3 supplements to improve symptoms of ADHD are still needed. At this point in time, eating foods high in omega-3 fatty acids is a reasonable approach for someone with $\mathrm{ADHD}^{9,10}$.

\section{Blood pressure}

The prevention of blood clotting, reduced blood pressure and a stabilize heart rhythm have all benefited from taking Omega-3 fish oil supplements ${ }^{11}$. One of the best ways to help prevent and treat heart disease is to eat a low-fat diet and to replace foods rich in saturated and trans-fat with those that are rich in monounsaturated and polyunsaturated fats (including omega- 3 fatty acids) ${ }^{12}$. Evidence suggests that EPA and DHA found in fish oil help reduce risk factors for heart disease including high cholesterol and high blood pressure. There is also strong evidence that these substances can help prevent and treat atherosclerosis by inhibiting the development of plaque and blood clots, each of which tends to clog arteries. Studies of heart attack survivors have found that daily omega-3 fatty acid supplements dramatically reduce the risk of death, subsequent heart attacks, and stroke. Similarly, people who eat an ALA-rich diet are less likely to suffer a fatal heart attack ${ }^{13}$.

\section{Heart attack}

Large amount of medical literature testifies that Omega 3 fish oil supplements can prevent heart attacks ${ }^{14}$.

\section{Stroke}

Strong evidence from population-based studies suggests that omega-3 fatty acid intake (primarily from fish), helps protect against stroke caused by plaque buildup and blood clots in the arteries that lead to the brain ${ }^{15}$. In fact, eating at least two servings of fish per week can reduce the risk of stroke by as much as $50 \%$. However, people who eat more than three grams of omega- 3 fatty acids per day (equivalent to 3 servings of fish per day) may be at an increased risk for hemorrhagic stroke, a potentially fatal type of stroke in which an artery in the brain leaks or ruptures ${ }^{16}$.

\section{Rheumatoid arthritis}

Most clinical studies investigating the use of omega-3 fatty acid supplements for inflammatory joint conditions have focused almost entirely on rheumatoid arthritis ${ }^{17}$. Several articles reviewing the research in this area conclude that omega-3 fatty acid supplements reduce tenderness in joints, decrease morning stiffness, and allow for a reduction in the amount of medication needed for people with rheumatoid arthritis. In addition, laboratory studies suggest that diets rich in omega-3 fatty acids (and low in omega- 6 fatty acids) may benefit people with other inflammatory disorders, such as osteoarthritis ${ }^{18}$. In fact, several test tube studies of cartilage-containing cells have found that omega- 3 fatty acids decrease inflammation and reduce the activity of enzymes that destroy cartilage ${ }^{18}$. Similarly, New Zealand green lipped mussel (Perna canaliculus) ${ }^{19},{ }^{20}$, another potential source of omega-3 fatty acids, has been shown to reduce joint stiffness and pain, increase grip strength, and enhance walking pace in a small group of people with osteoarthritis. In some participants, symptoms worsened before they improved. 


\section{Osteoporosis}

Studies suggest that omega-3 fatty acids such as EPA help increase levels of calcium in the body, deposit calcium in the bones, and improve bone strength ${ }^{21}$. In addition, studies also suggest that people who are deficient in certain essential fatty acids (particularly EPA and gamma-linolenic acid [GLA], an omega-6 fatty acid) are more likely to suffer from bone loss than those with normal levels of these fatty acids. In a study of women over 65 with osteoporosis, those given EPA and GLA supplements experienced significantly less bone loss over three years than those who were given a placebo ${ }^{22}$. Many of these women also experienced an increase in bone density.

\section{Diabetes}

People with diabetes tend to have high triglyceride and low HDL levels. Omega-3 fatty acids from fish oil can help lower triglycerides and raise HDL, so people with diabetes may benefit from eating foods or taking supplements that contain DHA and EPA. ALA (from flaxseed, for example) may not have the same benefit as DHA and EPA because some people with diabetes lack the ability to efficiently convert ALA to a form of omega-3 fatty acids that the body can use readily ${ }^{23}$.

\section{Weight loss}

Many people who are overweight suffer from poor blood sugar control, diabetes, and high cholesterol. Studies suggest that overweight people who follow a weight loss program including exercise tend to achieve better control over their blood sugar and cholesterol levels when fish rich in omega-3 fatty acids (such as salmon, mackerel, and herring) is a staple in their low fat $\operatorname{diet}^{24}$.

\section{Eating disorders}

Studies suggest that men and women with anorexia nervosa have lower than optimal levels of polyunsaturated fatty acids (including ALA and GLA). To prevent the complications associated with essential fatty acid deficiencies, some experts recommend that treatment programs for anorexia nervosa include PUFArich foods such as fish and organ meats (which include omega-6 fatty acids) $)^{25}$.

\section{Menstrual pain}

In a study of nearly 200 Danish women, those with the highest dietary intake of omega-3 fatty acids had the mildest symptoms during menstruation ${ }^{26}$.

\section{Inflammatory bowel disease (IBD)}

When added to medication, such as sulfasalazine (a standard medication for IBD), omega-3 fatty acids may reduce symptoms of Crohn's disease and ulcerative colitis - the two types of $\mathrm{IBD}^{27}$. More studies to investigate this preliminary finding are under way. In animals, it appears that ALA works better at decreasing bowel inflammation than EPA and $\mathrm{DHA}^{28}$. Plus, fish oil supplements can cause side effects that are similar to symptoms of IBD (such as flatulence and diarrhoea). Time-release preparations may help reduce these unwanted effects ${ }^{29}$.

\section{Colon cancer}

Consuming significant amounts of foods rich in omega-3 fatty acids appears to reduce the risk of colorectal cancer. For example, Eskimos, who tend to follow a high fat diet but eat significant amounts of fish rich in omega- 3 fatty acids, have a low rate of colorectal cancer. Animal studies and laboratory studies have found that omega-3 fatty acids prevent worsening of colon cancer while omega- 6 fatty acids promote the growth of colon tumours ${ }^{30}$. Daily consumption of EPA and DHA also appeared to slow or even reverse the progression of colon cancer in people with early stages of the disease ${ }^{31}$.

However, in an animal study of rats with metastatic colon cancer $^{32}$ (in other words, cancer that has spread to other parts of the body such as the liver), omega-3 fatty acids actually promoted the growth of cancer cells in the liver. Until more information is available, it is best for people with advanced stages of colorectal cancer to avoid omega-3 fatty acid supplements and diets rich in this substance ${ }^{32}$.

\section{Breast cancer}

Although not all experts agree, women who regularly consume foods rich in omega-3 fatty acids over many years may be less likely to develop breast cancer. In addition, the risk of dying from breast cancer may be significantly less for those who eat large quantities of omega-3 from fish and brown kelp seaweed (common in Japan). This is particularly true among women who substitute fish for meat. The balance between omega-3 and omega- 6 fatty acids appears to play an important role in the development and growth of breast cancer. Further research is still needed to understand the effect that omega-3 fatty acids may have on the prevention or treatment of breast cancer. For example, several researchers speculate that omega-3 fatty acids in combination with other nutrients (namely, vitamin $\mathrm{C}$, vitamin E, beta-carotene, selenium, and coenzyme Q10) may prove to be of particular value for preventing and treating breast cancer ${ }^{33}$.

\section{Prostate cancer}

Laboratory and animal studies indicate ${ }^{34,35}$ that omega-3 fatty acids (specifically, DHA and EPA) may inhibit the growth of prostate cancer. Similarly, population based studies of groups of men suggest that a low-fat diet with 
the addition of omega-3 fatty acids from fish or fish oil help prevent the development of prostate cancer ${ }^{36}$. Like breast cancer, the balance of omega-3 to omega- 6 fatty acids appears to be particularly important for reducing the risk of this condition ${ }^{37}$. ALA, however, may not offer the same benefits as EPA and DHA. In fact, one recent study evaluating 67 men with prostate cancer found that they had higher levels of ALA compared to men without prostate cancer. More research in this area is needed.

\section{Skin disorders}

In one study, 13 people with a particular sensitivity to the sun known as photo dermatitis showed significantly less sensitivity to UV rays after taking fish oil supplements ${ }^{38}$. Still, research indicates that ${ }^{38}$ ) topical sunscreens are much better at protecting the skin from damaging effects of the sun than omega-3 fatty acids. In another study of 40 people with psoriasis, those who were treated with medications and EPA supplements did better than those treated with the medications alone ${ }^{39}$. In addition, many clinicians believe that flaxseed (which contains omega-3 fatty acids) is helpful for treating acne.

\section{Burns}

Essential fatty acids have been used to reduce inflammation and promote wound healing in burn victims. Animal research indicates that omega-3 fatty acids help promote a healthy balance of proteins in the body-protein balance is important for recovery after sustaining a burn ${ }^{40}$. Further research is necessary to determine whether omega $3 \mathrm{~s}$ benefit people in the same way.

\section{Macular degeneration}

A questionnaire administered to more than 3,000 people over the age of 49 found that those who consumed more fish in their diet were less likely to have macular degeneration (a serious age-related eye condition that can progress to blindness) than those who consumed less fish $^{41}$. Similarly, a study comparing 350 people with macular degeneration to 500 without found that those with a healthy dietary balance of omega-3 and omega- 6 fatty acids and higher intake of fish in their diets were less likely to have this particular eye disorder. Another larger study confirms that EPA and DHA from fish, four or more times per week, may reduce the risk of developing macular degeneration ${ }^{42}$, ${ }^{43}$. Notably, however, this same study suggests that ALA may actually increase the risk of this eye condition.

\footnotetext{
Asthma

Preliminary research suggests that omega-3 fatty acid supplements (in the form of perilla seed oil, which is
}

rich in ALA) may decrease inflammation and improve lung function in adults with asthma. Omega 3 fish oil supplements of 3.3 grams a day can reduce breathing difficulties with asthma ${ }^{44}$.Omega- 6 fatty acids have the opposite effect: they tend to increase inflammation and worsen respiratory function ${ }^{45}$. In a small, well-designed study of 29 children with asthma, those who took fish oil supplements rich in EPA and DHA for 10 months had improvement in their symptoms compared to children who took a placebo pill ${ }^{44}$.

\section{Drug interactions \\ Aspirin}

Omega-3 fatty acids may increase the blood-thinning effects of aspirin or Warfarin. The combination of aspirin and omega-3 fatty acids may be helpful under certain circumstances (such as heart disease), they should only be taken together under the guidance and supervision of doctor ${ }^{46}$.

\section{Cyclosporine}

Omega-3 fatty acids supplements during cyclosporine therapy may reduce toxic side effects (such as high blood pressure and kidney damage) associated with this medication in transplant patients ${ }^{47,48}$.

\section{Cholesterol-lowering medications}

Following certain nutritional guidelines, including increasing the amount of omega- 3 fatty acids in the diet and reducing the omega- 6 to omega- 3 ratio, may allow a group of cholesterol lowering medications known as "statins" to work more effectively ${ }^{49}$.

\section{Nonsteroidal Anti-inflammatory drugs (NSAIDs)}

In an animal study ${ }^{50}$, treatment with omega-3 fatty acids reduced the risk of ulcers from Nonsteroidal antiinflammatory drugs (NSAIDs). More research is needed to evaluate whether omega-3 fatty acids would have the same effects in people.

\section{Conclusion}

Omega Oil's excellent levels of precursor raw material fatty acids Alpha-linolenic acid (ALA) - Omega 3 and the precursor linoleic acid (LA) - Omega 6, make it an ideal supplement for those whose lifestyle makes them suffer the consequences of deficiency in taking of fatty acid products that are essential for a smooth running metabolism. Our body requires an ideal balance between Omega $3 \& 6$ to maintain its correct functioning. Although further research is needed, preliminary evidence suggests that omega-3 fatty acids may also prove helpful in protecting against certain infections and treating a variety of conditions including ulcers, migraine headaches, preterm labour, emphysema, psoriasis, glaucoma, Lyme disease. 


\section{References}

1. Bracco U, Deckelbaum RJ. Polyunsaturated Fatty Acids in Human Nutrition. New York: Raven Press; 1992.

2. Giles H, Pilditch CA. Effects of mussel (Perna Canaliculus) biodeposit decomposition on benthic respiration and nutrient fluxes. Marine Biology.2006:150 (2):261 -71.

3. Su KP, Huang SY, Chiu CC, Shen WW. Omega-3 fatty acids in major depressive disorder. A preliminary double-blind, placebocontrolled trial. Eur Neuropsychopharmacol. $2003 ; 13(4): 267-71$

4. Raine A. Schizotypal Personality: Neurodevelopmental and Psychosocial Trajectories. Annual Review of Clinical Psychology. April 2006; Vol. 2, 291-326.

5. Stoll AL, Severus WE, Freeman MP, et al. Omega 3 fatty acids in bipolar disorder: a preliminary double-blind placebo-controlled trial. Arch Gen Psychiatry. 1999:56(5):407-12.

6. Laugharne JD, Mellor JE, Peet M. Fatty acids and schizophrenia. Lipids. 1996; 31(Suppl): S-163-5.

7. Bruinsma KA, Taren DL. Dieting, essential fatty acid intake and depression. Nutrition Rev. 2000; 58(4):98-108.

8. Macrae F. Fish oil 'calms children better than Ritalin’. Daily Mail. 20 June 2006.

9. Burgess J, Stevens L, Zhang W, Peck L. Longchain polyunsaturated fatty acids in children with attention-deficit hyperactivity disorder. Am J Clin Nutr. 2000; 71(Suppl):327S-30S.

10. Arnold LE, Kleykamp D, Votolato N, Gibson RA, Horrocks L. Potential link between dietary intake of fatty acid and behavior: pilot exploration of serum lipids in attentiondeficit hyperactivity disorder. J Child Adolesc Psychopharmacol. 1994; 4(3):171-82.

11. Appel LJ. Nonpharmacologic therapies that reduce blood pressure: a fresh perspective. Clin Cardiol. 1999; 22(Suppl. III):III1-III5.

12. Angerer P, von Schacky C. n-3 polyunsaturated fatty acids and the cardiovascular system. Curr Opin Lipidol. 2000; 11(1):57-63.

13. Albert CM, Hennekens CH, O'Donnell CJ, et al. Fish consumption and risk of sudden cardiac death. JAMA. 1998; 279(1):23-28.

14. Harper CR, Jacobson TA. The fats of life: the role of omega-3 fatty acids in the prevention of coronary heart disease. Arch Intern Med. 2001; 161(18):2185-92.
15. Mori, Trevor A, Beilin, Lawrence J. Longchain omega 3 fatty acids, blood lipids and cardiovascular risk reduction. Current Opinion in Lipidology. February 2001;12(1):11-7.

16. Iso $\mathrm{H}$, Rexrode $\mathrm{KM}$, Stampfer MJ, Manson JE, et al. Intake of fish and omega- 3 fatty acids and risk of stroke in women. JAMA. 2001; 285(3):304-12.

17. Danao-Camara TC, Shintani TT. The dietary treatment of inflammatory arthritis: case reports and review of the literature. Hawaii Med J. 1999; 58(5):126-31.

18. Curtis CL, Hughes CE, Flannery CR, et al. N-3 fatty acids specifically modulate catabolic factors involved in articular cartilage degradation. J Biol Chem. 2000; 275(2):721-4.

19. Gibson SL, Gibson RG. The treatment of arthritis with a lipid extract of Perna canaliculus: a randomized trial. Complement Ther Med. 1998; 6:122-6.

20. Halpern G-M. Anti-inflammatory effects of a stabilized lipid extract of Perna canaliculus (Lyprinol). Allerg Immunol (Paris). 2000; 32(7):272-8.

21. Kruger MC, Coetzer H, de Winter R, Gericke G. Calcium, gamma-linolenic acid and eicosapentaenoic acid supplementation in senile osteoporosis. Aging Clin Exp Res. 1998; 10:385-94.

22. Kruger MC, Horrobin DF. Calcium metabolism, osteoporosis and essential fatty acids: a review. Prog Lipid Res. 1997; 36:131-151.

23. Friedberg CE, Janssen MJ, Heine RJ, Grobbee DE. Fish oil and glycemic control in diabetes: a meta-analysis. Diabetes Care. 1998; 21:494500.

24. Mori TA, Bao, DQ, Burke V, et al. Dietary fish as a major component of a weight-loss diet: effect on serum lipids, glucose, and insulin metabolism in overweight hypertensive subjects. Am J Clin Nutr. 1999; 70:817-25.

25. Holman RT, Adams CE, Nelson RA, et al. Patients with anorexia nervosa demonstrate deficiencies of selected essential fatty acids, compensatory changes in nonessential fatty acids and decreased fluidity of plasma lipids. J Nutr. 1995;125:901-7.

26. Deutch B. Menstrual pain in Danish women correlated with low n-3 polyunsaturated fatty acid intake. Eur J Clin Nutr. 1995; 49(7):50816. 
27. Belluzzi A, Boschi S, Brignola C, Munarini A, Cariani C, Miglio F. Polyunsaturated fatty acids and inflammatory bowel disease. Am J Clin Nutr. 2000; 71:339S-42S.

28. Dichi I, Frenhane P, Dichi JB, et al. Comparison of omega-3 fatty acids and sulfasalazine in ulcerative colitis. Nutrition. 2000; 16:87-90.

29. Belluzzi A, Brignolia C, Campieri M, et al. Effect of an enteric-coated fish-oil preparation on relapses in Crohn's disease. New Engl J Med. 1996; 334(24):1558-60.

30. Klurfeld DM, Bull AW. Fatty acids and colon cancer in experimental models. Am J Clin Nut. 1997; 66:1530S-8S.

31. Anti M, Armelau F, Marra G, et al. Effects of different doses of fish oil on rectal cell proliferation in patients with sporadic colonic adenomas. Gastroenterology. 1994; 107(6):1892-4.

32. Griffini P, Fehres O, Klieverik L, et al. Dietary omega-3 polyunsaturated fatty acids promote colon carcinoma metastasis in rat liver. Can Res. 1998; 58(15):3312-9.

33. Lockwood K, Moesgaard S, Hanioka T, Folkers $\mathrm{K}$. Apparent partial remission of breast cancer in 'high risk' patients supplemented with nutritional antioxidants, essential fatty acids, and coenzyme Q10. Mol Aspects Med. 1994; 15:231-40.

34. Freeman VL, Meydani M, Yong S, Pyle J, Flanigan RC, Waters WB, Wojcik EM. Prostatic levels of fatty acids and the histopathology of localized prostate cancer. J Urol. 2000;164(6):2168-2172.

35. Berquin IM, Min $\mathrm{Y}, \mathrm{Wu}{ }^{\mathrm{R}}$ et al. Modulation of prostate cancer genetic risk by omega-3 and omega-6 fatty acids. J. Clin. Invest. 2007;117(7): 1866-75.

36. Newcomer LM, King IB, WicklundKG, Stanford JL. The association of fatty acids with prostate cancer risk. Prostate. 2001; 47(4):262-8.

37. Aronson WJ, Glaspy JA, Reddy ST, Reese D, Heber D, Bagga D. Modulation of omega-3/ omega- 6 polyunsaturated ratios with dietary fish oils in men with prostate cancer. Urology. 2001; 58(2):283-8

38. Ando H, Ryu A, Hashimoto A, Oka M, Ichihashi M. Linoleic acid and alpha-linolenic acid lightens ultraviolet-induced hyper pigmentation of the skin. Arch Dermatol Res. 1998; 290(7):375-381.

39. Danno K, Sugie N. Combination therapy with low-dose etretinate and eicosapentaenoic acid for psoriasis vulgaris. J Dermatol. 1998; 25:703-5.
40. De-Souza DA, Greene LJ. Pharmacological nutrition after burn injury. J Nutr. 1998; 128:797-803.

41. Cho E, Hung S, Willet WC, et al. Prospective study of dietary fat and the risk of age-related macular degeneration. Am J Clin Nutr. 2001;73(2):209-18

42. Seddon JM, Rosner B, Sperduto RD, et al. Dietary fat and risk for advanced age-related macular degeneration. Arch Opthalmol. 2001; 119(8):1191-9.

43. Soyland E, Funk J, Rajka G, Sandberg M, et al. Effect of dietary supplementation with very-long chain n-3 fatty acids in patients with psoriasis. N Engl J Med. 1993;328(25):1812-6.

44. Okamoto M, Misunobu F, Ashida K, et al. Effects of dietary supplementation with n-3 fatty acids compared with n- 6 fatty acids on bronchial asthma. Int Med. 2000; 39(2):10711.

45. Nagakura T, Matsuda S, Shichijyo K, Sugimoto H, Hata K. Dietary supplementation with fish oil rich in omega-3 polyunsaturated fatty acids in children with bronchial asthma. Eur Resp J. 2000;16(5):861-5.

46. Balk E, Chung M, Lichtenstein A, et al. Effects of Omega-3 Fatty Acids on Cardiovascular Risk Factors and Intermediate Markers of Cardiovascular Disease. Evidence Report/ Technology Assessment No. 93.

47. Badalamenti S, Salerno F, Lorenzano E, et al. Renal Effects of Dietary Supplementation with Fish Oil in Cyclosporine-Treated Liver Transplant Patients. Hepatol. 1995; 2(6):1695701.

48. van der Heide JJH, Bilo HJ, Tegzess AM, Donker AJ. The effects of dietary supplementation with fish oil on renal function in cyclosporine-treated renal transplant recipients. Transplantation. 1990; 49:523-527

49. Caron MF, White CM. Evaluation of the antihyperlipidemic properties of dietary supplements. Pharmacotherapy. 2001; 21(4):481-7.

50. Al-Harbi MM, Islam MW, Al-Shabanah OA, AlGharably NM. Effect of acute administration of fish oil (omega-3 marine triglyceride) on gastric ulceration and secretion induced by various ulcerogenic and necrotizing agents in rats. Fed Chem Toxic. 1995;33(7):555-8. 